\title{
Transphalangeal Toe Amputation
}

\author{
Jamal J. Hoballah
}

\section{Indication}

- Toe gangrene/chronic osteomyelitis

\section{Essential Steps}

1. Noninvasive vascular testing suggesting healing of toe amputation is likely.

2. Perform an elliptical skin incision perpendicular to the axis of the toe.

3. Elevate the periosteum to the level of the amputation.

4. Divide the bone.

5. Close the skin without tension.

\section{Complications}

- Nonhealing amputation site

- Infection

\section{Template Operative Dictation}

\section{Preoperative Diagnosis Toe gangrene/infection}

Procedure Toe amputation

J.J. Hoballah, MD, MBA, FACS

Department of Surgery, American University of

Beirut Medical Center, Beirut, Lebanon

e-mail: jh34@aub.edu.lb

\section{Postoperative Diagnosis Same}

Indications This is a __year-old malelfemale with a gangrenous/infected toe. Noninvasive testing with toe pressure and transcutaneous oxygen tension suggested good skin perfusion. The risks and benefits of surgical intervention were discussed with the patient, and he/she elected to undergo the procedure.

Description of Procedure Time-outs were performed using both preinduction and pre-incision safety checklists to verify correct patient, procedure, site, and additional critical information prior to beginning the procedure. The procedure was performed under regional/general anesthesia. The right/left foot was prepped and draped in a sterile fashion. A skin incision was then performed at the level of the mid-proximal phalynx. The incision was deepened to the level of the bone. The periosteum was then elevated with a periosteal elevator. The bone was then transected at the proximal phalangeal level, $1.5 \mathrm{~cm}$ proximal to the level of the skin incision. Hemostasis was then secured and the wound was irrigated. The skin was then closed with interrupted sutures of 4-0 nylon.

A debriefing checklist was completed to share information critical to postoperative care of the patient.

The patient tolerated the procedure well and was taken to the postanesthesia care unit in stable condition. 